Annals of Pure and Applied Mathematics

Vol. 14, No. 2, 2017, 237-243

ISSN: 2279-087X (P), 2279-0888(online)

Published on 31 August 2017

www.researchmathsci.org

DOI: http://dx.doi.org/10.22457/apam.v14n2a5

Annals of

Pure and Applied

Mathematics

\title{
Cone S-Metric Space and Fixed Point Theorems of Contractive Mappings
}

\author{
D.Dhamodharan $^{1}$ and R. Krishnakumar ${ }^{2}$ \\ ${ }^{1}$ Department of Mathematics, Jamal Mohamed College (Autonomous) \\ Tiruchirappalli-620020, India. E-mail: dharan_raj28@yahoo.co.in \\ ${ }^{2}$ Department of Mathematics, Urumu Dhanalakshmi College \\ Tiruchirappalli-620019, India \\ E-mail address: srksacet@yahoo.co.in \\ ${ }^{1}$ Corresponding author
}

Received 28 July 2017; accepted 19 August 2017

Abstract. In this paper, we discuss the concept of generalized $S$-Metric space of Cone $S$ Metric space and prove some contractive conditions of unique fixed point.

Keywords: Common fixed point, cone $\mathrm{S}$ metric space, cone normed space cone $\mathrm{C}$-class function.

AMS Mathematics Subject Classification (2010): 47H10, 54H25

\section{Introduction and mathematical preliminaries}

In 2007, Huang and Zhang [10] introduced the concept of cone metric spaces and fixed point theorems of contraction mappings; Any mapping $T$ of a complete cone metric space $X$ into itself that satisfies, for some $0 \leq k<1$, the inequality $d(T x, T y) \leq k d(x, y)$ $\forall x, y \in X$ has a unique fixed point. In 2012, Sedghi et al. [6] introduced the concept of generalization of fixed point theorems in $S$-metric spaces. Rahman and Sarwar [7] are discussed in fixed point results of Altman integral type mappings in $S$-metric space. In recently, Ozgur and Tas [8] are discuss new contractive conditions of integral type on complete $S$-metric spaces. In 2017, Gholidahneh, et al. [12] are introduce the notion of integral type contractive mapping with respect to ordered s-metric spaces and coupled common fixed point theorems of integral type contraction.

In this paper, we discuss the concept of cone $S$ metric space for some contraction of fixed point theorems.

Definition1.1. [10] Let $E$ be a Banach space. A subset $P$ of $E$ is called a cone if and only if:

1. $\quad P$ is closed, nonempty and $P \neq 0$

2. $a x+b y \in P$ for all $x, y \in P$ and nonnegative real numbers $a, b$

3. $P \cap(-P)=\{0\}$.

Given a cone $P \subset E$, we define a partial ordering $\preccurlyeq$ with respect to $\mathrm{P}$ by $x \leqslant y$ if and only if $y-x \in P$. We will write $x<y$ to indicate that $x \leqslant y$ but $x \neq y$, while $x, y$ 


\section{D.Dhamodharan and R. Krishnakumar}

will stand for $y-x \in$ int $P$, where int $P$ denotes the interior of $P$. The cone $P$ is called normal if there is a number $K>0$ such that $0 \preccurlyeq x \leqslant y$ implies $\|x\| \preccurlyeq K|| y \mid$ for all $x, y \in E$. The least positive number satisfying the above is called the normal constant.

The cone $P$ is called regular if every increasing sequence which is bounded from above is convergent. That is, if $\left\{x_{n}\right\}$ is sequence such that $x_{1} \preccurlyeq x_{2} \preccurlyeq \cdots \preccurlyeq x_{n} \cdots \preccurlyeq y$ for some $y \in E$, then there is $x \in E$ such that $\left\|x_{n}-x\right\| \rightarrow 0$ as $n \rightarrow 0$. Equivalently the cone $P$ is regular if and only if every decreasing sequence which is bounded from below is convergent. It is well known that a regular cone is a normal cone. Suppose $E$ is a Banach space, $P$ is a cone in $E$ with int $P \neq 0$ and $\preccurlyeq$ is partial ordering with respect to $P$.

Example 1.2. [4] Let $K>1$ be given. Consider the real vector space with

with supremum norm and the cone

$$
E=\left\{a x+b: a, b \in R ; x \in\left[1-\frac{1}{k}, 1\right]\right\}
$$

in $E$. The cone $P$ is regular and so normal.

$$
P=\{a x+b: a \geq 0, b \geq 0\}
$$

Definition 1.3. [10] Let $X$ be a nonempty set. Suppose the mapping $d: X \times X \rightarrow E$ satisfies

1. $d(x, y) \geq 0$, and $d(x, y)=0$ if and only if $x=y \forall x, y \in X$,

2. $d(x, y)=d(y, x), \forall x, y \in X$,

3. $d(x, y) \leq d(x, z)+d(z, y), \forall x, y, z \in X$,

Then $(X, d)$ is called a cone metric space simply CMS.

Lemma 1.4. [11] Every regular cone is normal.

Example 1.5. [10] Let $E=R^{2}$

$X=R$ and $d: X \times X \rightarrow E$ such that

$$
P=\{(x, y): x, y \geq 0\}
$$

$$
d(x, y)=(|x-y|, \alpha|x-y|)
$$

where $\alpha \geq 0$ is a constant. Then $(X, d)$ is a Cone metric space.

Definition 1.6. [9] Let $X \neq \emptyset$ be any set and $S: X \times X \times X \rightarrow[0, \infty)$ be a function satisfying the following conditions for all $u, v, z, a \in X$.

1. $S(u, v, z) \geq 0$

2. $S(u, v, z)=0$ if and only if $u=v=z$.

3. $\quad S(u, v, z) \leq S(u, u, a)+S(v, v, a)+S(z, z, a)$

Then the function $\mathrm{S}$ is called an $\mathrm{S}$-metric on $\mathrm{X}$ and the pair $(X, S)$ is called an $S$-metric space simply SMS.

Example 1.7. [12] Let $X$ be a non empty set, $d$ is ordinary metric space on $X$, then $S(x, y, z)=d(x, z)+d(y, z)$ is an $S$ - metric on $X$.

Definition 1.8. Suppose that $E$ is a real Banach space, then $P$ is a cone in $E$ with int $P \neq \varnothing$, and $\preccurlyeq$ is partial ordering with respect to $P$. Let $X$ be a nonempty set, a function 
Cone S-Metric Space and Fixed Point Theorems of Contractive Mappings

$d: X \times X \times X \rightarrow E$ is called a cone $S$ metric on $X$ if it satisfies the following conditions with

1. $S(u, v, z) \geqslant 0$

2. $S(u, v, z)=0$ if and only if $u=v=z$.

3. $S(u, v, z) \leqslant S(u, u, a)+S(v, v, a)+S(z, z, a)$

Then the function $\mathrm{S}$ is called an cone $\mathrm{S}$-metric on $\mathrm{X}$ and the pair $(X, S)$ is called an cone $S$-metric space simply CSMS.

Example 1.9. Let $E=R^{2}, P=\{(x, y): x, y \geqslant 0\}, X=R$ and $d: X \times X \times X \rightarrow E$ such that then $S(x, y, z)=(d(x, z)+d(y, z), \alpha(d(x, z)+d(y, z))),(\alpha>0)$ is an cone $S$ metric on $X$.

Lemma 1.10. Let $(X, S)$ be an cone $S$-metric space. Then we have $S(u, u, v)=$ $S(v, v, u)$.

Definition 1.11. Let $(X, S)$ be an cone $S$-metric space .

1. A sequence $\left\{u_{n}\right\}$ in $X$ converges to $u$ if and only if $S\left(u_{n}, u_{n}, u\right) \rightarrow 0$ as $n \rightarrow \infty$. That is, there exists $n_{0} \in N$ such that for all $n \geqslant n_{0}, S\left(u_{n}, u_{n}, u\right) \ll c$ for each $c \in E, 0 \ll c$. We denote this by $\lim _{n \rightarrow \infty} u_{n}=u$ or $\lim _{n \rightarrow \infty} S\left(u_{n}, u_{n}, u\right)=0$.

2. A sequence $\left\{u_{n}\right\}$ in $X$ is called a Cauchy sequence if $S\left(u_{n}, u_{n}, u_{m}\right) \rightarrow 0$ as $n, m \rightarrow$ $\infty$. That is, there exists $n_{0} \in N$ such that for all $n, m \geqslant n_{0}, S\left(u_{n}, u_{n}, u_{m}\right) \ll c$ for each $c \in E, 0 \ll c$.

3. The cone $S$-metric space $(X, S)$ is called complete if every Cauchy sequence is convergent.

In the following lemma we see the relationship between a cone metric and an cone Smetric.

Lemma 1.12. Let $(X, d)$ be a cone metric space. Then the following properties are satisfied:

1. $S(u, v, z)=d(u, z)+d(v, z)$ for all $u, v, z \in X$ is an cone S-metric on X.

2. $u_{n} \rightarrow u$ in $\{X, d\}$ if and only if $u_{n} \rightarrow u$ in $\left(X, S_{d}\right)$ :

3. $\left\{u_{n}\right\}$ is Cauchy in $\{X, d\}$ if and only if $\left\{u_{n}\right\}$ is Cauchy in $\left(X, S_{d}\right)$ :

4. $\{X, d\}$ is complete if and only if $\left(X, S_{d}\right)$ is complete.

\section{Main result}

Theorem 2.1. Let $(X, S)$ be a complete cone $S$-metric space and $P$ be a normal cone with normal constant $K$. Suppose the mapping $T: X \rightarrow X$ satisfies the following conditions:

$$
S(T u, T u, T v) \preccurlyeq h S(u, u, v)
$$

for all $u, v \in X$. Then $T$ has a unique fixed point $w \in X$ and we have $\lim _{n \rightarrow \infty} T^{n} u=w$, for each $u \in X$

Proof: Let $u_{0} \in X$ and the sequence $\left\{u_{n}\right\}$ be defined as $T^{n} u_{0}=u_{n}$. Suppose that $u_{n} \neq u_{n+1}$ for all $n$. Using the inequality (2.1), we obtain

$$
S\left(u_{n}, u_{n}, u_{n+1}\right) \leqslant h S\left(u_{n-1}, u_{n-1}, u_{n}\right) \leqslant \cdots \leqslant h^{n} S\left(u_{0}, u_{0}, u_{1}\right)
$$

If we take limit for $n \rightarrow \infty$, using the inequality (2.2) we get

$$
\lim _{n \rightarrow \infty} S\left(u_{n}, u_{n}, u_{n+1}\right)=0 \text {, }
$$




\section{D.Dhamodharan and R. Krishnakumar}

Since $h \in(0,1)$. The $\varepsilon>0$ implies $\lim _{n \rightarrow \infty} S\left(u_{n}, u_{n}, u_{n+1}\right)=0$.

Now we show that the sequence $\left\{u_{n}\right\}$ is a Cauchy sequence.Assume that $\left\{u_{n}\right\}$ is not Cauchy. Then there exists an $0 \ll \varepsilon$ and subsequences $\left\{m_{k}\right\}$ and $\left\{n_{k}\right\}$ such that $m_{k} \prec$ $n_{k} \prec m_{k+1}$ with

And

$$
S\left(u_{m_{k}}, u_{m_{k}}, u_{n_{k}}\right) \geqslant \epsilon
$$

$$
S\left(u_{m_{k}}, u_{m_{k}}, u_{n_{k-1}}\right) \prec \varepsilon
$$

Hence using Lemma (1.10), we have

And

$$
\begin{gathered}
S\left(u_{m_{k-1}}, u_{m_{k-1}}, u_{n_{k-1}}\right) \leqslant 2 S\left(u_{m_{k-1}}, u_{m_{k-1}}, u_{m_{k}}\right)+S\left(u_{n_{k-1}}, u_{n_{k-1}}, u_{m_{k}}\right) \\
\prec 2 S\left(u_{m_{k-1}}, u_{m_{k-1}}, u_{m_{k}}\right)+\epsilon
\end{gathered}
$$

$$
\lim _{k \rightarrow \infty} S\left(u_{m_{k}}-1, u_{m_{k}}-1, u_{m_{k}}-1\right) \preccurlyeq \epsilon
$$

Using the inequalities (2.1), (2.3) and (2.5), we obtain

$$
\epsilon \preccurlyeq S\left(u_{m_{k}}, u_{m_{k}}, u_{n_{k}}\right) \preccurlyeq S\left(u_{m_{k}}-1, u_{m_{k}}-1, u_{n_{k}}-1\right) \preccurlyeq h \epsilon
$$

which is a contradiction with our assumption since $h \in(0,1)$. So the sequence $\left\{u_{n}\right\}$ is Cauchy. Using the completeness hypothesis, there exists $w \in X$ such that $\lim _{n \rightarrow \infty} T^{n} u_{0}=w$. From the inequality (2.1) we find

$\begin{aligned} S\left(T w, T w, u_{n}+1\right) & =S\left(T w, T w, u_{n}\right) \preccurlyeq \\ \text { Therefore, } \lim _{n \rightarrow 0}|| S\left(T w, T w, u_{n}+1\right)|| & \leqslant h K|| S(T w, T w, w)||\end{aligned}$

$$
S\left(T w, T w, u_{n}+1\right)=S\left(T w, T w, u_{n}\right) \preccurlyeq h S\left(w, w, u_{n}\right)
$$

$$
\lim _{n \rightarrow 0}|| S(T w, T w, w)|| \preccurlyeq h K|| S(T w, T w, w)||
$$

Since $h \in(0,1)$, then $S(T w, T w, w)=0$ which is implies that $S(T w, T w, w) \ll 0$ thus $T w=w$.

To prove $T$ has unique fixed point.

Let $w, w_{1}$ be two fixed points of $T$ such that $w \neq w_{1}$. Taking $u=w$ and $v=w_{1}$ in equation (2.1) we have

$$
S\left(w, w, w_{1}\right)=S\left(T w, T w, w_{1}\right) \preccurlyeq h S\left(w, w, w_{1}\right)
$$

where $h \in(0,1)$, which is implies

$$
S\left(w, w, w_{1}\right)=0
$$

Thus $w=w_{1}$.

Corollary 2.2. Let $(X, S)$ be a complete cone $S$-metric space and $P$ be a normal cone with normal constant $K$. Suppose the mapping $T: X \rightarrow X$ satisfies the following conditions:

$$
S(T u, T u, T v) \preccurlyeq h \quad S(u, u, v)
$$

for all $u, v \in X$. Then $T$ has a unique fixed point $w \in X$ and we have $\lim _{n \rightarrow \infty} T^{n} u=w$, for each $u \in X$.

Theorem 2.3. Let $(X, S)$ be a complete cone $S$-metric space and $P$ be a normal cone with normal constant $K$. Suppose the mapping $T: X \rightarrow X$ satisfies the following conditions: $S(T u, T u, T v) \preccurlyeq h_{1} S(u, u, v)+h_{2} S(T u, T u, v)+h_{3} S(T v, T v, u)+$ $h_{4} \max \{S(T u, T u, v), S(T v, T v, u)\}$ for all $u, v \in X$ with non negative real numbers $h_{i}\left(i \in\{1,2,3,4)\right.$ satisfyingmax $\left\{h_{1}+\right.$ $\left.3 h_{3}+2 h_{4}, h_{1}+h_{2}+h_{3}\right\} \prec 1$. 
Cone S-Metric Space and Fixed Point Theorems of Contractive Mappings Then $T$ has a unique fixed point $w \in X$ and we have $\lim _{n \rightarrow \infty} T^{n} u=w$, for each $u \in X$. Proof: Let $u_{0} \in X$ and the sequence $\left\{u_{n}\right\}$ be defined as $\lim _{n \rightarrow \infty} T^{n} u_{0}=u_{n}$ Suppose that $u_{n} \neq u_{n+1}$ for all $n$. Using the inequality (2.7), the condition (S3) and Lemma (1.10), we get

$$
\begin{gathered}
S\left(u_{n}, u_{n}, u_{n+1}\right)=S\left(T u_{n-1}, T u_{n-1}, T u_{n}\right) \\
\preccurlyeq h_{1} S\left(u_{n-1}, u_{n-1}, u_{n}\right)+h_{2} S\left(u_{n}, u_{n}, u_{n}\right)+h_{3} S\left(u_{n+1}, u_{n+1}, u_{n-1}\right) \\
\quad+h_{4} \max \left\{S\left(u_{n}, u_{n}, u_{n-1}\right), S\left(u_{n+1}, u_{n+1}, u_{n}\right)\right\} \\
=h_{1} S\left(u_{n-1}, u_{n-1}, u_{n}\right)+h_{3} S\left(u_{n+1}, u_{n+1}, u_{n-1}\right) \\
\quad+h_{4} \max \left\{S\left(u_{n}, u_{n}, u_{n-1}\right), S\left(u_{n+1}, u_{n+1}, u_{n}\right)\right\} \\
\preccurlyeq h_{1} S\left(u_{n-1}, u_{n-1}, u_{n}\right)+h_{3}\left\{2 S\left(u_{n+1}, u_{n+1}, u_{n}\right)+h_{3} S\left(u_{n-1}, u_{n-1}, u_{n}\right)\right. \\
+h_{4} S\left(u_{n+1}, u_{n+1}, u_{n-1}\right)+h_{4} S\left(u_{n+1}, u_{n+1}, u_{n}\right) \\
=\left(h_{1}+h_{3}+h_{4}\right)+h_{4} S\left(u_{n-1}, u_{n-1}, u_{n}\right)+\left(2 h_{3}+h_{4}\right)+h_{4} S\left(u_{n}, u_{n}, u_{n+1}\right)
\end{gathered}
$$

which implies

$$
\mathrm{S}\left(u_{n}, u_{n}, u_{n+1}\right) \preccurlyeq \frac{h_{1}+h_{3}+h_{4}}{1-2 h_{3}-h_{4}} S\left(u_{n-1}, u_{n-1}, u_{n}\right)
$$

If we put $h=\frac{h_{1}+h_{3}+h_{4}}{1-2 h_{3}-h_{4}}$ then we find $h \prec 1$ since $h_{1}+3 h_{3}+2 h_{4} \prec 1$. Using the inequality (2.8), we have

$$
\lim _{n \rightarrow 0}\left\|S\left(u_{n}, u_{n}, u_{n+1}\right)\right\| \preccurlyeq h^{n} K\left\|S\left(u_{0}, u_{0}, u_{1}\right)\right\|(2.9)
$$

If we take limit for $n \rightarrow \infty$, using the inequality (2.9), we get

$$
\lim _{n \rightarrow \infty} S\left(u_{n}, u_{n}, u_{n+1}\right)=0
$$

since $h \in(0,1)$. For all $\varepsilon>0$ implies $\lim _{n \rightarrow \infty} S\left(u_{n}, u_{n}, u_{n+1}\right)=0$. By the similar arguments used in the proof of theorem (2.1), we see that the sequence $\left\{u_{n}\right\}$ is Cauchy. Then there exists $w \in X$ such that $\lim _{n \rightarrow \infty} T^{n} u_{0}=w$, since $(X, S)$ is a complete cone $S$-metric space. From the inequality (9) we find

$$
\begin{gathered}
S\left(u_{n}, u_{n}, T w\right)=S\left(T u_{n-1}, T u_{n-1}, T w\right) \\
\preccurlyeq h_{1} S\left(u_{n-1}, u_{n-1}, T w\right)+h_{2} S\left(u_{n}, u_{n}, w\right)+h_{3} S\left(T w, T w, u_{n-1}\right) \\
+h_{4} \max \left\{S\left(u_{n}, u_{n}, u_{n-1}\right), S(T w, T w, w)\right\}
\end{gathered}
$$

Therefore, $\lim _{n \rightarrow 0}|| S\left(u_{n}, u_{n}, T w\right)|| \preccurlyeq\left(h_{3}+h_{4}\right) K\|S(T w, T w, w)\|$

$$
\|S(T w, T w, w)\| \preccurlyeq\left(h_{3}+h_{4}\right) K\|S(T w, T w, w)\|,
$$

since $h_{3}+h_{4} \prec 1$. Then $S(T w, T w, w)=0$ which implies that $S(T w, T w, w) \ll 0$ thus $T w=w$.

Prove that $T$ has unique fixed point. Let $w, w_{1}$ be two fixed points of $T$ such that $w \neq w_{1}$. Taking $u=w$ and $v=w_{1}$ in equation (2.7), we have

$$
S\left(w, w, w_{1}\right)=S\left(T w, T w, w_{1}\right)
$$

$\preccurlyeq h_{1} S\left(w, w, w_{1}\right)+h_{2} S\left(w, w, w_{1}\right)+h_{3} S\left(w_{1}, w_{1}, w\right)$

which implies

$$
+h_{4} \max \left\{S(w, w, w), S\left(w_{1}, w_{1}, w_{1}\right)\right\}
$$

Then we obtain

$$
S\left(w, w, w_{1}\right) \preccurlyeq\left(h_{1}+h_{2}+h_{3}\right) S\left(w, w, w_{1}\right)
$$

$S\left(w, w, w_{1}\right)=0$

that is, $w=w_{1}$ since $h_{1}+h_{2}+h_{3} \prec 1$. Consequently, $T$ has a unique fixed point $w \in X$. 


\section{D.Dhamodharan and R. Krishnakumar}

Corollary 2.4. Let $(X, S)$ be a complete cone $S$-metric space and $P$ be a normal cone with normal constant $K$.Suppose the mapping $T: X \rightarrow X$ satisfies the following conditions:

$$
\begin{gathered}
S(T u, T u, T v) \preccurlyeq h_{1} S(u, u, v)+h_{2} S(T u, T u, v)+h_{3} S(T v, T v, u)+ \\
h_{4} \max \{S(T u, T u, v), S(T v, T v, u)\}
\end{gathered}
$$

for all $u, v \in X$ with non-negative real numbers $h_{i}\left(i \in\{1,2,3,4)\right.$ satisfying $\max \left\{h_{1}+\right.$ $\left.3 h_{3}+2 h_{4}, h_{1}+h_{2}+h_{3}\right\} \prec 1$, Then $T$ has a unique fixed point $w \in X$ and we have $\lim _{n \rightarrow \infty} T^{n} u=w$, for each $u \in X$.

Theorem 2.5. Let $(X, S)$ be a complete cone $S$-metric space and $P$ be a normal cone with normal constant $K$. Suppose the mapping $T: X \rightarrow X$ satisfies the following conditions:

$S(T u, T u, T v) \preccurlyeq h_{1} S(u, u, v)+h_{2} S(T u, T u, u)+h_{3} S(T u, T u, v)+h_{4} S(T v, T v, u)$ $+h_{5} S(T v, T v, v)+h_{6} \max \{S(u, u, v), S(T u, T u, u), \mathrm{S}(T u, T u, v), S(T v, T v, u)$,

$S(T v, T v, v)\}$

for all $u, v \in X$ with non negative real numbers $h_{i}\left(i \in\{1,2,3,4,5,6)\right.$ satisfying $\max \left\{h_{1}+\right.$ $\left.h_{2}+3 h_{4}+h_{5}+3 h_{6}, h_{1}+h_{3}+h_{4}+h_{6}\right\} \prec 1$ Then $T$ has a unique fixed point $w \in X$ and we have $\lim _{n \rightarrow \infty} T^{n} u=w$, for each $u \in X$.

Proof: Let $u_{0} \in X$ and the sequence $\left\{u_{n}\right\}$ be defined as $\lim _{n \rightarrow \infty} T^{n} u_{0}=u_{n}$ Suppose that $u_{n} \neq u_{n+1}$ for all $n$. Using the inequality (2.7) the condition (S3) and Lemma (2.10) we get

$S\left(u_{n}, u_{n}, u_{n+1}\right)=S\left(T u_{n-1}, T u_{n-1}, T u_{n}\right)$

$\preccurlyeq h_{1} S\left(u_{n-1}, u_{n-1}, u_{n}\right)+h_{2} S\left(u_{n}, u_{n}, u_{n-1}\right)+h_{3} S\left(u_{n}, u_{n}, u_{n}\right)$ $+h_{4} S\left(u_{n+1}, u_{n+1}, u_{n-1}\right)+h_{5} S\left(u_{n+1}, u_{n+1}, u_{n}\right)$

$+h_{6} \max \left\{S\left(u_{n-1}, u_{n-1}, u_{n}\right), S\left(u_{n}, u_{n}, u_{n-1}\right), S\left(u_{n}, u_{n}, u_{n}\right), S\left(u_{n+1}, u_{n+1}, u_{n-1}\right)\right.$,

$\left.S\left(u_{n+1}, u_{n+1}, u_{n}\right)\right\}$

$\preccurlyeq\left(h_{1}+h_{2}+h_{4}+h_{6}\right) S\left(u_{n-1}, u_{n-1}, u_{n}\right)+\left(2 h_{4}+h_{5}+2 h_{6}\right) S\left(u_{n+1}, u_{n+1}, u_{n}\right)$

which implies

$S\left(u_{n}, u_{n}, u_{n}+1\right) \preccurlyeq\left(\frac{h_{1}+h_{2}+h_{4}+h_{6}}{1-2 h_{4}-h_{5}-2 h_{6}}\right) S\left(u_{n-1}, u_{n-1}, u_{n}\right)$

If we put $h=\frac{h_{1}+h_{2}+h_{4}+h_{6}}{1-2 h_{4}-h_{5}-2 h_{6}}$ then we find $h \preccurlyeq 1$ since $h_{1}+h_{2}+3 h_{4}+h_{5}+3 h_{6} \prec 1$.

Using the inequality (13) we have

$$
\lim _{n \rightarrow \infty}|| S\left(u_{n}, u_{n}, u_{n+1}\right)|| \preccurlyeq h^{n} K \| S\left(u_{0}, u_{0}, u_{1}\right) \mid
$$

If we take limit for $n \rightarrow \infty$, using the inequality (2.12), we get

$$
\lim _{n \rightarrow 0} S\left(u_{n}, u_{n}, u_{n+1}\right)=0
$$

since $h \in(0,1)$ The $\varepsilon>0$ implies $\lim _{n \rightarrow \infty} S\left(u_{n}, u_{n}, u_{n+1}\right)=0$ By the similar arguments used in the proof of Theorem (2.4), we see that the sequence $\left\{u_{n}\right\}$ is Cauchy. Then there exists $w \in X$ such that $\lim _{n \rightarrow \infty} T^{n} u_{0}=w$, since $(X, S)$ is a complete cone $S$-metric space. From the inequality (2.12) we find

$$
\begin{aligned}
& \qquad S\left(u_{n}, u_{n}, T w\right)=S\left(T u_{n}-1, T u_{n}-1, T w\right) \\
& \preccurlyeq h_{1} S\left(u_{n-1}, u_{n-1}, w\right)+h_{2} S\left(u_{n}, u_{n}, u_{n-1}\right)+h_{3} S\left(u_{n}, u_{n}, w\right) \\
& +h_{4} S\left(T w, T w, u_{n-1}\right)+h_{5} S(T w, T w, w) \\
& +h_{6} \max \left\{S\left(u_{n-1}, u_{n-1}, w\right), S\left(u_{n}, u_{n}, u_{n-1}\right), S\left(u_{n}, u_{n}, w\right), S\left(T w, T w, u_{n-1}\right), S(T w, T w, w)\right\} \\
& \text { Therefore, } \lim _{n \rightarrow 0}|| S\left(u_{n}, u_{n}, T w\right)|| \preccurlyeq\left(h_{4}+h_{5}+h_{6}\right) K \| S(T w, T w, w \|,
\end{aligned}
$$


Cone S-Metric Space and Fixed Point Theorems of Contractive Mappings

since $h_{4}+h_{5}+h_{6} \prec 1$. Then $S(T w, T w, w)=0$ which implies that $S(T w, T w, w) \ll 0$ thus $T w=w$.

Prove that $T$ has unique fixed point. Let $w, w_{1}$ be two fixed points of $T$ such that $w \neq w_{1}$. Taking $u=w$ and $v=w_{1}$ in equation (2.10), we have

$$
\begin{gathered}
S\left(w, w, w_{1}\right)=S\left(T w, T w, T w_{1}\right) \\
\leqslant h_{1} S\left(w, w, w_{1}\right)+h_{2} S(w, w, w)+h_{3} S\left(w, w, w_{1}\right)+h_{4} S\left(w_{1}, w_{1}, w\right) \\
+h_{5} S\left(w_{1}, w_{1}, w_{1}\right) \\
+h_{6} \max \left\{S\left(w, w, w_{1}\right), S(w, w, w), S\left(w, w, w_{1}\right), S\left(w_{1}, w_{1}, w\right), S\left(w_{1}, w_{1}, w_{1}\right)\right\}
\end{gathered}
$$

which implies

Then we obtain

$$
S\left(w, w, w_{1}\right) \preccurlyeq\left(h_{1}+h_{3}+h_{4}+h_{6}\right) S\left(w, w, w_{1}\right)
$$

$S\left(w, w, w_{1}\right)=0$,

that is, $w=w_{1}$ since $h_{1}+h_{3}+h_{4}+h_{6} \prec 1$. Consequently, $T$ has a unique fixed point $w \in X$.

\section{REFERENCES}

1. A.H.Ansari, Note on $\varphi-\psi$-contractive type mappings and related fixed point, The 2nd Regional Conference on Mathematics and Applications, PNU, September (2014) 377-380.

2. A.H.Ansari, S.Chandok, N.Hussin and L.Paunovic, Fixed points of $(\psi, \phi)$ - weak contractions in regular cone metric spaces via new function, J. Adv. Math. Stud., 9(1) (2016) 72-82.

3. M.S.Khan, M.Swaleh and S.Sessa, Fixed point theorems by altering distances between the points, Bulletin of the Australian Math. Society, 30 (1) (1984) 1-9.

4. R.Krishnakumar and D.Dhamodharan, Fixed point theorems in normal cone metric space, International J. of Math. Sci. Engg. Appls., 10(III) (2016) 213-224.

5. A.Gupta, Cyclic contraction on cone S-metric space, International journal of Analysis and Applications, 3(2) (2013) 119-130.

6. N.Y.Ozgur and N.Tas, New contractive conditions of integral type on complete Smetric space s, Math. Sci., DOI10.1007/s40096-017-0226-0.

7. S.Sedghi, N.Shobe and A.Aliouche, A generalization of fixed point theorems in Smetric spaces, Mat. Vesn., 64(3) (2012) 258-266.

8. M.U.Rahman and M.Sarwar, Fixed point results of Altman integral type mappings in S-metric spaces, Int. J. Anal. Appl., 10(1) (2016) 58-63.

9. N.Y.Ozgur and N.Tas, Some fixed point theorems on S-metric spaces, Mat. Vesnik, 69(1) (2017) 39-52.

10. H.Long-Guang and Z.Xian, Cone metric spaces and fixed point theorems of contractive mappings, J. Math. Anal. Appl., 332 (2007) 1468-1476.

11. Sh. Rezapour and R.Hamlbarani, Some notes on the paper' cone metric spaces and fixed point theorems of contractive mappings, J. Math. Anal. Appl., 345 (2008) 719724.

12. N.Tas and N.Yilmaz, Ozgur new generalized fixed point results on sb-metric spaces, arxiv: 1703.01868v2 [math.gn] 17 apr 2017.

13. A.Gholidahneh, S.Sedghi, T.Došenovic and S.Radenovic, Ordered S-metric spaces and coupled commonfixed point theorems of integral type contraction, Mathematics Interdisciplinary Research, 2 (2017) $71-84$. 